'Departamento de Nefrología, Escuela de Medicina, Facultad de Medicina Pontificia Universidad Católica de Chile. Santiago, Chile. 2Escuela de Medicina, Facultad de Medicina Pontificia Universidad Católica de Chile. Santiago, Chile. anterno de Medicina.

Trabajo no recibió financiamiento. Los autores declaran no tener conflictos de interés.

Correspondencia: Dr. Rodrigo A. Sepúlveda Departamento de Nefrología. Diagonal Paraguay 362, piso 4. Santiago, Chile. rrsepulveda@uc.cl

\title{
Metahemoglobinemia, una entidad de diagnóstico complejo. Reporte de un caso
}

\author{
RODRIGO A. SEPÚLVEDA ${ }^{1}$, ESTEBAN BARNAFI ${ }^{2, a}$, \\ VICENTE ROJAS ${ }^{2, a}$, AQUILES JARA ${ }^{1}$
}

\section{Methemoglobinemia caused by dapsone. Report of one case}

(Rev Med Chile 2020; 148: 1838-1843) Blue.

Key words: Blood Gas Analysis; Dapsone; Methemoglobinemia; Methylene

\begin{abstract}
Methemoglobinemia is a rare condition with serious consequences if not diagnosed. We report the case of a 64-year-old woman with a history of allergy to sulfa drugs and a recent diagnosis of a small vessel vasculitis (ANCA-p) who started induction therapy with corticosteroids and rituximab. Due to the need for infectious prophylaxis, and considering her history, dapsone was administered instead of cotrimoxazole after ruling out glucose-6-phosphate dehydrogenase deficiency. During the admission to the hospital for her second dose of rituximab, and while being asymptomatic, she persistently presented a pulse oximetry $<90 \%$ despite the administration of $\mathrm{O}_{2}$. Therefore, the infusion was postponed to study the patient. The arterial gasometric study by direct potentiometry revealed an $\mathrm{O}_{2}$ saturation of $98 \%$, with a saturation gap $>5 \%$. Considering the use of dapsone, a methemoglobinemia was suspected and confirmed by co-oximetry (methemoglobinemia 9\%). Dapsone was suspended and one week later, her methemoglobinemia was absent.
\end{abstract}


Se inició tratamiento con corticoides más rituximab. Evoluciona de manera favorable, clínicamente asintomática y con normalización de los parámetros inflamatorios. Por la inmunosupresión más corticoterapia se agregó omeprazol, calcio-vitamina D y dapsona. Esta última en reemplazo de cotrimoxazol, por antecedente de alergia a sulfas. Previo a iniciar dapsona, se evaluó la actividad de la enzima glucosa-6-fosfato deshidrogenasa (G6PDH) que resultó en 18 minutos (normal: 10-20 min).

La paciente ingresó estable y clínicamente asintomática para su segunda dosis de rituximab. Bien perfundida, sin palidez, con piel y mucosas rosadas, sin alteraciones respiratorias y sin infecciones intercurrentes. En el control de signos vitales se objetiva: temperatura $36^{\circ} \mathrm{C}$, presión arterial 116/76 $\mathrm{mmHg}$, frecuencia cardiaca 74 $\mathrm{lpm}$, frecuencia respiratoria $18 \mathrm{rpm}$ y oximetría de pulso con saturación de $86 \%$. Se administró $\mathrm{O}_{2}$ por naricera a $3 \mathrm{~L} / \mathrm{m}$. Pese a registrar oximetría de pulso en varios sitios y con distintas máquinas, el valor siempre resultaba alterado $(<90 \%)$.

Por los antecedentes de la paciente se decidió postergar la infusión de rituximab y estudiar con una nueva tomografía computada de tórax, que no mostraba cambios respecto a estudios previos. Gases arteriales mediante potenciometría directa: $\mathrm{pH} 7,46, \mathrm{HCO}_{3}-27 \mathrm{mEq} / \mathrm{L}, \mathrm{PaCO}_{2} 38$ $\mathrm{mmHg}, \mathrm{PaO}_{2} 101 \mathrm{mmHg}$, saturación arterial de $\mathrm{O}_{2}\left(\mathrm{SaO}_{2}\right) 99 \%$.

Por el uso de dapsona y gap de saturación, se sospecha presencia de metahemoglobinemia, la cual es confirmada mediante CO-oximetría por espectofotometría; resultando metahemoglobina $9 \%$ (normal $0-1,5 \%$ ) y carboxihemoglobina $1,7 \%$ (normal $<5 \%$ ).

Con estos hallazgos, se suspendió la dapsona y la paciente fue hospitalizada 1 semana después para recibir la dosis de rituximab. En esa oportunidad se controló la metahemoglobinemia que resultó $0 \%$.

Se obtuvo el consentimiento de la paciente para reportar el caso.

\section{Discusión}

En el humano, la hemoglobina $(\mathrm{Hb})$ presenta una cinética que permite entregar eficientemente el oxígeno a las células. Sin embargo, pese a su cinética favorable, la hemoglobina normalmente viaja saturada de $\mathrm{O}_{2}$. Esto significa que, por mucho que aumente el oxígeno disuelto en sangre (medido como $\mathrm{PaO}_{2}$ ), no incrementará el contenido de oxígeno.

La hemoglobina (HB) se une al oxígeno gracias a que posee 4 moléculas de fierro. Para que esta unión se lleve a cabo, el fierro debe estar reducido (ferroso o $\mathrm{Fe}^{+2}$ ), ya que en estado oxidado (férrico $\mathrm{o} \mathrm{Fe}^{+3}$ ) no ocurre. Si la hemoglobina porta alguna de sus moléculas de fierro en estado $\mathrm{Fe}^{+3}$, el resto $\left(\mathrm{Fe}^{+2}\right)$ aumenta su afinidad por el $\mathrm{O}_{2}{ }^{1}$. Es decir, la hemoglobina con fierro oxidado transporta menos oxígeno y no lo entrega a los tejidos ${ }^{2,3}$.

La hemoglobina que porta fierro oxidado se denomina metahemoglobina (metaHb). Normalmente, se produce de manera espontánea a velocidad de $3 \%$ de la $\mathrm{Hb}$ circulante al día ${ }^{1}$. Afortunadamente, el eritrocito tiene una maquinaria enzimática que reduce la $\mathrm{Hb}$ oxidada manteniendo los niveles normales de metaHb en sangre $<1 \%{ }^{3}$.

Quien reduce la metaHb para devolver el fierro a su estado ferroso es el glutatión reducido. A su vez, el glutatión oxidado adquiere su poder reductor gracias a moléculas de NADPH. Estas últimas se producen mediante la generación de pentosas, a partir de la glucosa, en una reacción que involucra a la enzima G6PDH (Figura 1). La glicólisis anaeróbica, también, genera moléculas de alto poder reductor (NADH). Normalmente, estas se utilizan en la mitocondria para producir energía, sin embargo, el eritrocito (que carece de mitocondrias), desvía todo este poder reductor hacia la conversión de $\mathrm{Hb}$ oxidada a reducida, para poder transportar $\mathrm{O}_{2}$ eficientemente a los tejidos. La reducción de metaHb, a partir de $\mathrm{NADH}$, se realiza mediante la enzima citocromo-b5 reductasa (Cb5R) (Figura 1).

Para que exista un exceso de metahemoglobina en sangre, debe estar alterada su reducción o existir una sobreproducción de esta. Los defectos en la reducción de metaHb generalmente son trastornos genéticos, tales como déficit de enzimas G6PDH y Cb5R. La sobreproducción de metaHb se observa cuando existen condiciones "oxidantes" $(\text { Tabla } 1)^{3-6}$.

Clínicamente, la cianosis se hace evidente con una metahemoglobinemia de $8-12 \%$, pero genera síntomas recién con niveles $>15 \%{ }^{4}$. Los primeros síntomas son: cefalea, debilidad, fatiga, aturdimiento, mareo, ansiedad. Posteriormente, 


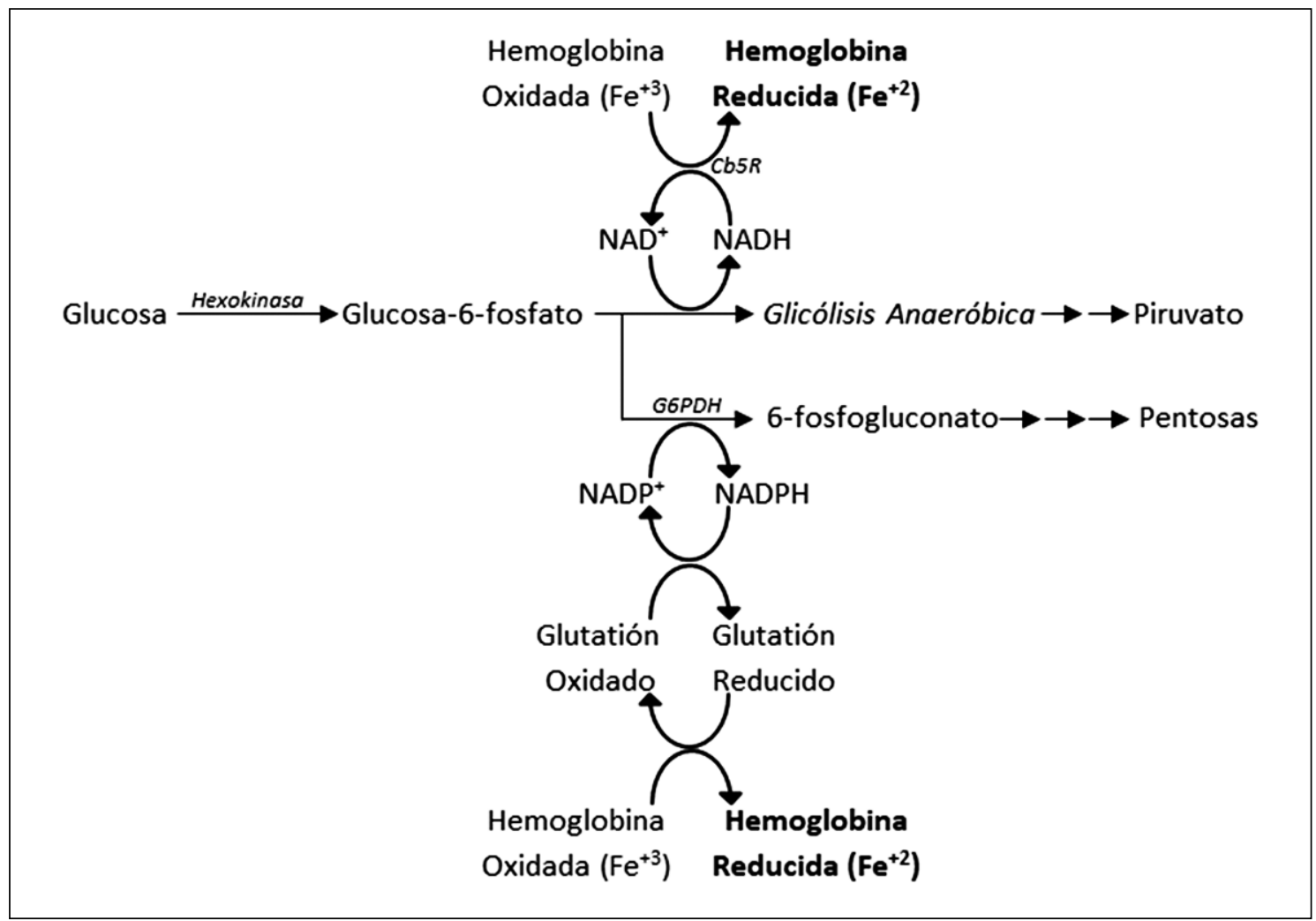

Figura 1.

aparecen alteraciones derivadas de hipoxia: taquicardia, taquipnea, disnea, compromiso de conciencia, síncope, arritmias, disfunción orgánica, insuficiencia respiratoria y acidosis láctica ${ }^{7}$ Una metahemoglobinemia grave $(>60 \%)$ se asocia a coma, convulsiones y muerte ${ }^{1,3,8}$.

Además del cuadro clínico, el primer acercamiento a un paciente con metahemoglobinemia será una oximetría de pulso alterada. Este equipo emite 2 longitudes de onda (660 y $940 \mathrm{~nm}$ ) que atravesarán un objeto y se leerá la absorbancia de sustancias pulsátiles (sangre arterial). La capacidad de una molécula de "absorber" una longitud de onda se denomina "absorbancia". La oxihemoglobina absorbe preferentemente la onda de $940 \mathrm{~nm}$; en cambio, la desoxihemoglobina, la onda de $660 \mathrm{~nm}$. En una razón entre absorbancias $(660 \mathrm{~nm} / 940 \mathrm{~nm})$, un menor resultado indicará más oxihemoglobina. Así, esta razón es proporcional a la saturación de oxígeno de la hemoglobina (sin indicar cuál es su concentración) ${ }^{9}$. La metahemoglobina absorbe ambas longitudes de onda, pero preferentemente $660 \mathrm{~nm}$, por lo que simula un estado de desaturación. Sin embargo, sobre $35 \%$ de metahemoglobina se establece una meseta de $82-85 \%$ de saturación de oxígeno (en la oximetría de pulso), independizándose de los niveles de metahemoglobina ${ }^{3,10}$.

La CO-oximetría en sangre es una técnica que permite detectar directamente metahemoglobina, ya que emite varias longitudes de onda pudiendo reconocer: oxihemoglobina, desoxihemoglobina, metahemoglobina y carboxihemoglobina ${ }^{11}$.

La medición tradicional de gases arteriales en sangre cuantifica directamente $\mathrm{pH}, \mathrm{PaO}_{2}, \mathrm{PaCO}_{2}$ y concentración de $\mathrm{Hb}$. El bicarbonato, exceso de base y $\mathrm{SaO}_{2}$ corresponden a estimaciones a partir de los parámetros medidos. Para la $\mathrm{SaO}_{2}$ se asume un estado "normal" de la hemoglobina y su capacidad de unión a $\mathrm{O}_{2}$, calculándolo según el $\mathrm{O}_{2}$ disuelto en sangre $\left(\mathrm{PaO}_{2}\right)$ y concentración de $\mathrm{Hb}$. Por este motivo, la oxihemoglobina resulta "falsamente normalizada". Un gap de saturación $>5 \%$, entre la $\mathrm{SaO}_{2}$ obtenida desde gases arteriales 
Tabla 1. Condiciones oxidantes inductoras de metahemoglobinemia

\begin{tabular}{|c|c|}
\hline Drogas & $\begin{array}{l}\text { Azul de metileno (en deficiencia de G6PDH) } \\
\text { Benzocaína } \\
\text { Bupivacaína } \\
\text { Celecoxib } \\
\text { Cloroquina } \\
\text { Dapsona } \\
\text { Dinitrato de isosorbide } \\
\text { Fenacetina } \\
\text { Fenazopiridina } \\
\text { Fenitoína } \\
\text { Flutamida } \\
\text { Lidocaína } \\
\text { Menadiona } \\
\text { Metoclorpramida } \\
\text { Nitrato de plata } \\
\text { Nitroglicerina } \\
\text { Nitroprusiato } \\
\text { Popper (nitratos) } \\
\text { Prilocaína } \\
\text { Primaquina } \\
\text { Sulfas (sulfametoxazol) } \\
\text { Sulfonamidas } \\
\text { Zopiclona }\end{array}$ \\
\hline $\begin{array}{l}\text { Tóxicos y contaminantes } \\
\text { ambientales }\end{array}$ & $\begin{array}{l}\text { Acetanilida } \\
\text { Aguas o alimentos contaminados con nitratos } \\
\text { Aloxana } \\
\text { Anilinas } \\
\text { Arsano } \\
\text { Benceno y derivados } \\
\text { Bromatos } \\
\text { Cloratos } \\
\text { Fenilhidrazina } \\
\text { Fenol } \\
\text { Ferricianuro de potasio } \\
\text { Herbicidas (Diquat, Paraquat) } \\
\text { Hidroxilamina } \\
\text { Lubricantes para aumentar octanaje en gasolinas } \\
\text { Naftalina } \\
\text { Nitratos (nitroalcano, nitrofurano, nitrato de metilo, óxido nítrico, nitroetano, nitrobence- } \\
\text { no, nitrato de amilo, nitrato de butilo, nitrato isobutílico, nitrato de sodio, nitrato de plata, } \\
\text { dinitrofenol, dinitrotolueno) } \\
\text { Pesticidas } \\
\text { Sales de cobre } \\
\text { Trinitrotolueno } \\
\text { Veneno de Loxosceles gaucho }\end{array}$ \\
\hline Alimentos & Habas (vicina) \\
\hline Enfermedades sistémicas & $\begin{array}{l}\text { Acidosis metabólica grave } \\
\text { Infección intestinal por bacterias productoras de nitritos } \\
\text { Infección grave }\end{array}$ \\
\hline
\end{tabular}


versus la $\mathrm{SaO}_{2}$ entregada por oximetría de pulso, es sugerente de una metahemoglobinemia ${ }^{3,10}$.

El tratamiento en metahemoglobinemia inducida por drogas debe ser la suspensión del agente causal. Sin embargo, se debe administrar azul de metileno $1 \%$ en infusión IV a pacientes con niveles $>30 \%$ o $>20 \%$ si presentan síntomas (considerar valores más bajos en casos de anemia o enfermedad cardiopulmonar concomitante). Su acción permite convertir prontamente toda la hemoglobina oxidada a reducida por reacciones dependientes de $\mathrm{NADPH}^{12}$. Se administra $1-2 \mathrm{mg} / \mathrm{kg}$ durante 5 minutos, disminuyendo significativamente los niveles en una hora, siendo posible repetir la dosis en 30 minutos $^{3}$. Sin embargo, el azul de metileno puede ser deletéreo en déficit de G6PDH, ya que favorece la aparición de hemólisis ${ }^{13}$. Otro tratamiento es el ácido ascórbico, pero requiere altas dosis iv o tratamientos prolongados $\mathrm{vo}^{14}$. El azul de metileno es más útil en metahemoglobinemias agudas adquiridas, mientras que el ácido ascórbico vo puede emplearse en cuadros crónicos y genéticos.

En nuestra paciente, la causa de metahemoglobinemia fue la dapsona. Este fármaco oxidante tiene una capacidad de inducir metahemoglobinemia en forma dosis dependiente, siendo más vulnerables aquellos pacientes que tienen déficit en la actividad de la enzima G6PDH o anemia ${ }^{1,15}$. También puede producir hemólisis extravascular por estrés oxidativo eritrocitario grave ${ }^{16}$.

La dapsona se utiliza para el tratamiento y profilaxis de lepra, malaria, neumonía por $P$. jirovecii $y$ dermatitis herpetiforme, entre otros cuadros ${ }^{17}$. Tiene una vida media prolongada gracias a su circulación enterohepática, por lo que continúa presente en la sangre luego de su suspensión y sus niveles rebotan luego del tratamiento con azul de metileno ${ }^{3}$. Es la causa más frecuente de metahemoglobinemia adquirida, con $42 \%$ de los casos $^{18}$. La asociación de cimetidina al tratamiento con dapsona podría evitar la aparición de metahemoglobinemia ${ }^{15,17}$.

Nuestra paciente se encontraba asintomática, en otras series se describe al menos cianosis o disnea ${ }^{17,19}$. Fue el gap de saturación lo que permitió sospechar el diagnóstico. Como la metaHb era $<30 \%$, solo fue necesario suspender el fármaco para tratarla adecuadamente. No se utilizó azul de metileno ni ácido ascórbico. Algunos de los casos descritos en la literatura se complican con hemóli$\operatorname{sis}^{17}$, daño macular, falla renal aguda ${ }^{20}$ y neuropatía periférica $^{21}$. Afortunadamente, ninguna de estas complicaciones ocurrió en nuestra paciente.

\section{Referencias}

1. Umbreit J. Methemoglobin--it's not just blue: a concise review. Am J Hematol 2007; 82 (2): 134-44.

2. Mansouri A, Lurie AA. Concise review: methemoglobinemia. Am J Hematol 1993; 42 (1): 7-12.

3. Wright RO, Lewander WJ, Woolf AD. Methemoglobinemia: etiology, pharmacology, and clinical management. Ann Emerg Med 1999; 34 (5): 646-56.

4. Mokhlesi B, Leikin JB, Murray P, Corbridge TC. Adult toxicology in critical care: Part II: specific poisonings. Chest 2003; 123 (3): 897-922.

5. Kaushik P, Zuckerman SJ, Campo NJ, Banda VR, Hayes SD, Kaushik R. Celecoxib-induced methemoglobinemia. Ann Pharmacother 2004; 38 (10): 1635-8.

6. Fung HT, Lai CH, Wong OF, Lam KK, Kam CW. Two cases of methemoglobinemia following zopiclone ingestion. Clin Toxicol (Phila) 2008; 46 (2): 167-70.

7. Margulies DR, Manookian CM. Methemoglobinemia as a cause of respiratory failure. J Trauma 2002; 52 (4): 796-7.

8. Jaffé ER. Methemoglobin pathophysiology. Prog Clin Biol Res 1981; 51: 133-51.

9. Jubran A. Pulse oximetry. Crit Care 2015; 19 (1): 272.

10. Mokhlesi B, Leiken JB, Murray P, Corbridge TC. Adult toxicology in critical care: part I: general approach to the intoxicated patient. Chest 2003; 123 (2): 577-92.

11. Mathews PJ. Co-oximetry. Respir Care Clin N Am 1995; 1 (1): 47-68.

12. Jaffé ER, Neurmann G. A comparision of the effect of menadione, methylene blue and ascorbic acid on the reduction of methemoglobin in vivo. Nature 1964; 202: 607-8.

13. Rosen PJ, Johnson C, McGehee WG, Beutler E. Failure of methylene blue treatment in toxic methemoglobinemia. Association with glucose-6-phosphate dehydrogenase deficiency. Ann Intern Med 1971; 75 (1): 83-6.

14. Eder HA, Finch C, McKee RW. Congenital methemoglobinemia. A clinical and biochemical study of a case. J Clin Invest 1949; 28 (2): 265-72.

15. Coleman MD. Dapsone: modes of action, toxicity and possible strategies for increasing patient tolerance. Br J Dermatol 1993; 129 (5): 507-13.

16. Castillo RA, Hernández B, Sierra E, Rodríguez E, Arreguin R. Glucose-6-Phosphate Dehydrogenase: Update and Analysis of New Mutations around the World. Int J Mol Sci 2016; 17 (12): 2069.

17. Barclay JA, Ziemba SE, Ibrahim RB. Dapsone-induced 
methemoglobinemia: a primer for clinicians. Ann Pharmacother 2011; 45 (9): 1103-15.

18. Ash-Bernal R, Wise R, Wright SM. Acquired methemoglobinemia: a retrospective series of 138 cases at 2 teaching hospitals. Medicine (Baltimore) 2004; 83 (5): 265-73.

19. Burke P, Jahangir K, Kolber MR. Dapsone-induced methemoglobinemia: case of the blue lady. Can Fam
Physician 2013; 59 (9): 958-61.

20. Kenner DJ, Holt K, Agnello R, Chester GH. Permanent retinal damage following massive dapsone overdose. $\mathrm{Br}$ J Ophthalmol 1980; 64 (10): 741-4.

21. Abhayambika K, Chacko A, Mahadevan K, Najeeb OM. Peripheral neuropathy and haemolytic anaemia with cherry red spot on macula in dapsone poisoning. J Assoc Physicians India 1990; 38 (8): 564-5. 